\title{
Isobavachalcone inhibits Pseudorabies virus by impairing virus-induced cell-to-cell fusion
}

\author{
Yu Wang ${ }^{1,2}$, Tian-Xin Liư ${ }^{2}$ Tong-Yun Wang ${ }^{2}$, Yan-Dong Tang ${ }^{2^{*}}$ and Ping Wei ${ }^{1^{*}}$
}

\begin{abstract}
Pseudorabies virus (PRV) is an important pathogen that threatens the global swine industry. Currently, there is no effective drug that can clinically prevent or treat PRV infections. Isobavachalcone (IBC), a natural chalcone compound derived from Psoralea corylifolia, displays multiple biological activities, such as antibacterial, antifungal, and anticancer activities. Recently, it was found that IBC exhibited antiviral activity against an RNA virus, porcine reproductive and respiratory syndrome virus (PRRSV), in vitro. In the current study, we further demonstrated for the first time that IBC has a strong inhibitory effect on PRV. Through a viral luciferase expression assay, we showed that the inhibition step occurs mainly in the late stage of viral replication. Finally, via a cell-to-cell fusion assay, we demonstrated that IBC inhibits PRV by blocking virus-mediated cell fusion. Thus, IBC may be a candidate for further therapeutic evaluation against PRV infection in vivo.
\end{abstract}

Keywords: PRV, Isobavachalcone, Antiviral, Fusion

\section{Main text}

Pseudorabies virus (PRV) is a swine alphaherpesvirus that causes Aujeszky's disease in pigs $[1,2]$. PRV poses a serious threat to the pig industry, especially since novel PRV variants began emerging in 2011 [3, 4]. Most importantly, recent studies have reported that humans can be infected by PRV [5-7], indicating that PRV is also a potential threat to humans [6]. Thus, exploring new anti-PRV agents may be an effective means of controlling PRV. In this study, we explored the potential anti-PRV activity of isobavachalcone (IBC), a traditional Chinese medicine (TCM). The structure of IBC is shown in Fig. 1a. IBC was first isolated from Psoralea corylifolia in 1968 and possesses a broad spectrum of biological activities [8]. In a recent study, IBC also exhibited anti-porcine reproductive

\footnotetext{
*Correspondence: tangyandong2008@163.com; weiping@neau.edu.cn ${ }^{2}$ State Key Laboratory of Veterinary Biotechnology, Harbin Veterinary Research Institute of Chinese Academy of Agricultural Sciences, Harbin 150001, China

${ }^{1}$ College of Veterinary Medicine, Northeast Agricultural University, Harbin 150001, China
}

and respiratory syndrome virus (PRRSV) activity during the early stage of viral RNA synthesis [9]. To explore whether IBC has anti-PRV activity, we first evaluated the cytotoxicity of IBC on PK15 cells with a Cell Counting Kit-8 (CCK8, Dojindo Laboratories, Japan) according to the manufacturer's instructions. Cell viability was not changed relative to that of control cells at IBC concentrations of up to $25 \mu \mathrm{M}$. Next, we evaluated the antiviral activity of IBC via a recombinant PRV reporter virus expressing both enhanced green fluorescent protein (EGFP) and firefly luciferase [10]. These results showed that both EGFP expression and luciferase activity were significantly decreased in the IBC-treated group compared to the control group (Fig. 2a and b), indicating that PRV replication was significantly inhibited. However, the step in PRV replication that is influenced by IBC is unknown. To determine which step(s) in the viral life cycle are affected, we first treated PK15 cells with IBC and infected them $2 \mathrm{~h}$ later with the PRV reporter virus at an MOI of 0.01. IBC markedly inhibited PRV from 12 to $48 \mathrm{~h}$ post infection 


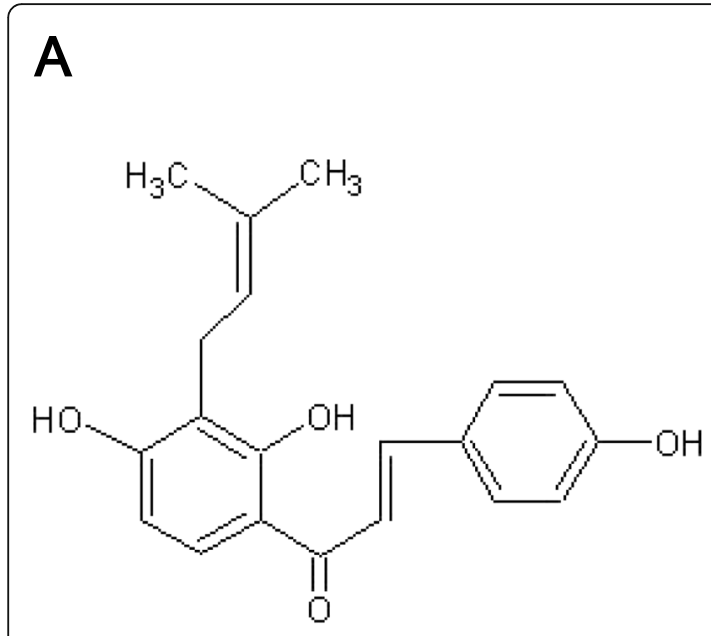

Isobavachalcone

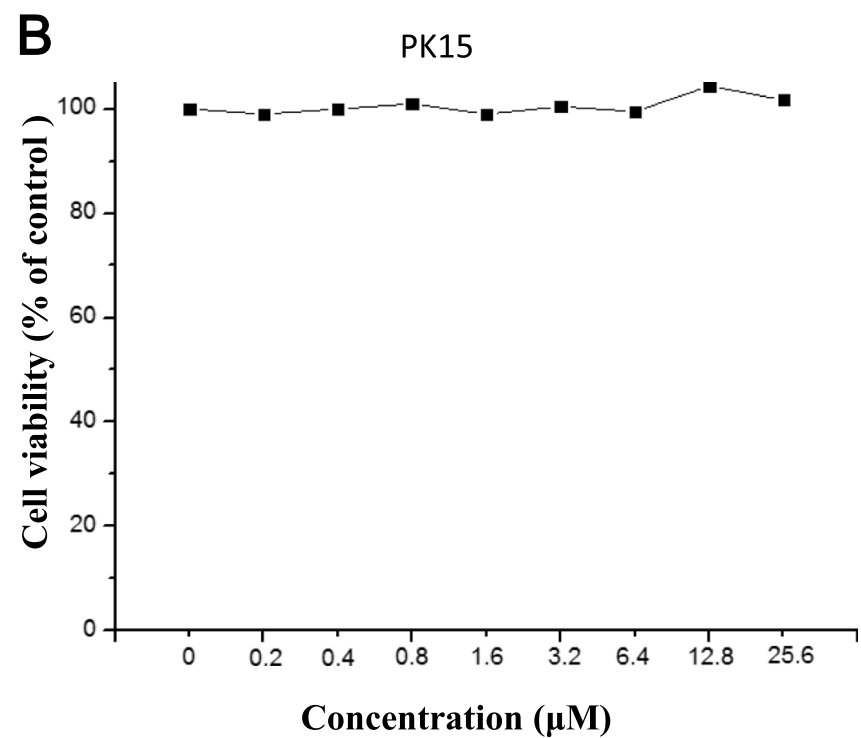

Fig. 1 IBC structure and cytotoxicity evaluation. a Chemical structure of IBC. b Cytotoxicity of IBC in PK15 cells incubated with different concentrations of IBC for $24 \mathrm{~h}$. The cell viability rate was assessed by measuring the absorbance at $490 \mathrm{~nm}$

(p.i.), whereas PRV replication was not influenced at 4 and 8 h p.i. (Fig. 3). According to the results of the one-step growth curves for the pseudorabies viruses, the virus titer reached a peak approximately $14 \mathrm{~h}$ post infection, so we recognized that 4 and $8 \mathrm{~h}$ post infection was the early stage. This pattern indicated that IBC inhibits PRV during the late stage of the viral lifecycle. In addition, these results demonstrated that the mechanisms by which IBC inhibits PRV and PRRSV may differ because IBC inhibited PRRSV at an early stage in our previous study [9]. PRV can induce cell-to-cell fusion, a very important step in viral spreading, at a late stage in the lifecycle. Here, to investigate whether IBC inhibits PRV replication at this stage, we performed transient transfection-based cell-to-cell fusion assays, as previously described [11, 12]. Briefly, RK13 cells were transfected with $200 \mathrm{ng}$ each of the EGFP expression plasmid (pDC315-EGFP [13]) or expression plasmids for the $\mathrm{gB}$, gL, and $\mathrm{gH}$ PRV glycoproteins (constructed in this study by subcloning these genes into the pCAGGS-HA vector) with Lipofectamine 2000 according to the manufacturer's instructions. Six hours later, the cell

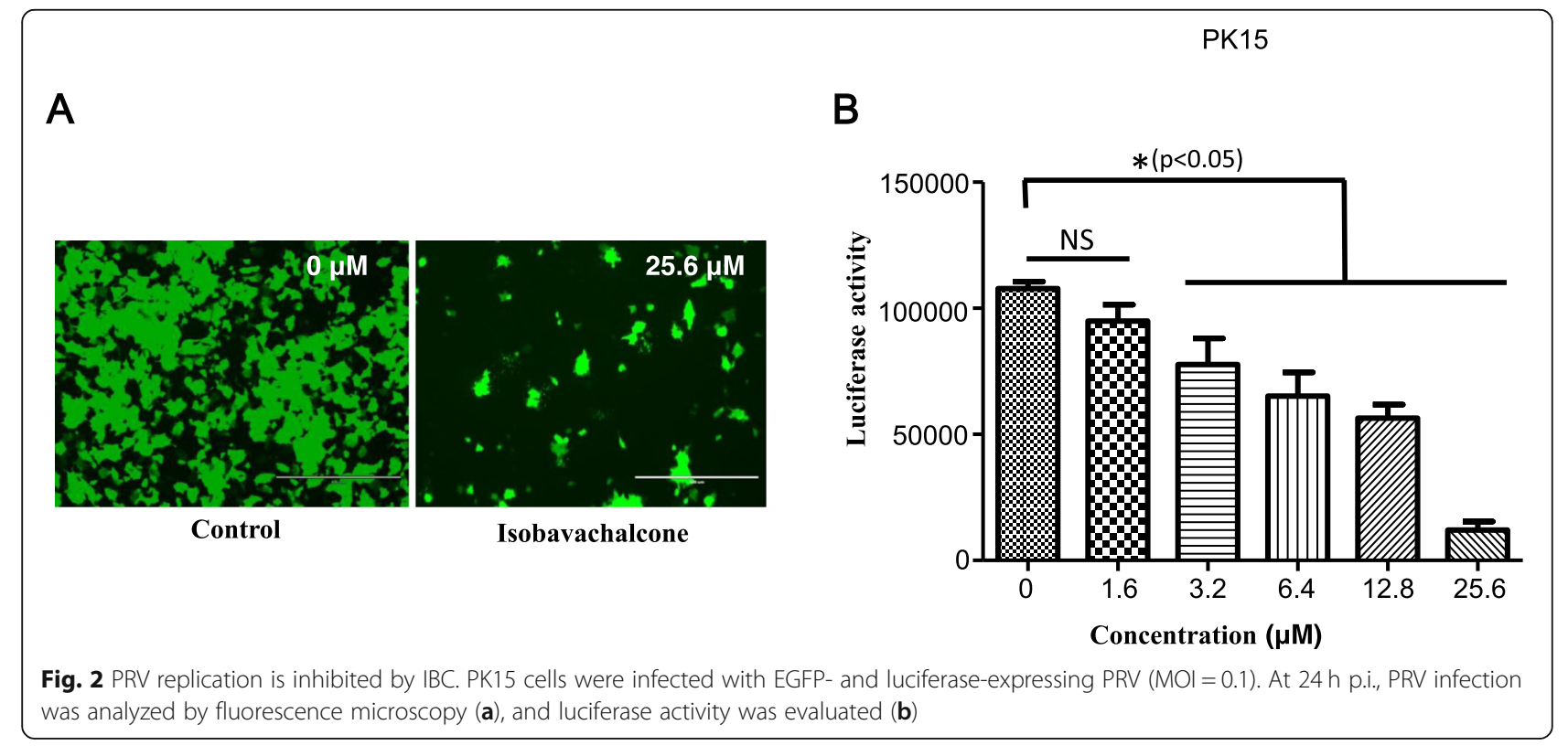




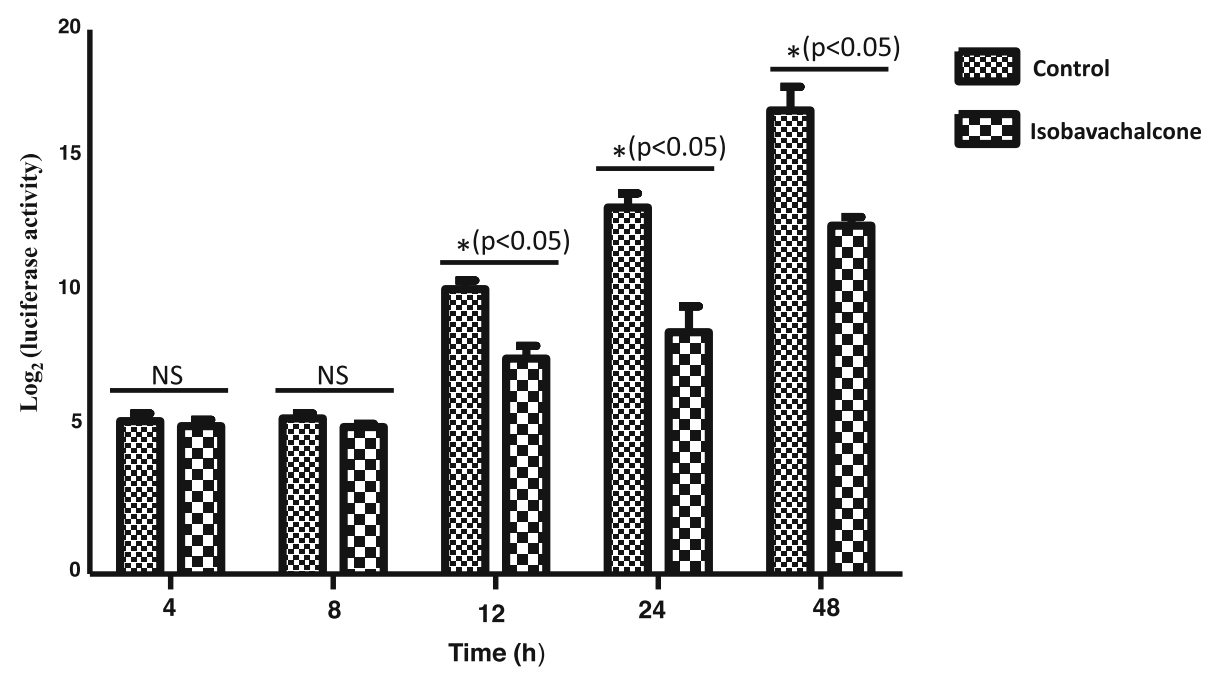

Fig. 3 IBC blocks the late stages of the PRV life cycle. PK15 cells were infected with PRV $(\mathrm{MOI}=0.1)$ and incubated in medium containing either ethanol or the indicated doses of IBC. Luciferase activity was evaluated at the indicated time points p.i

\section{A}
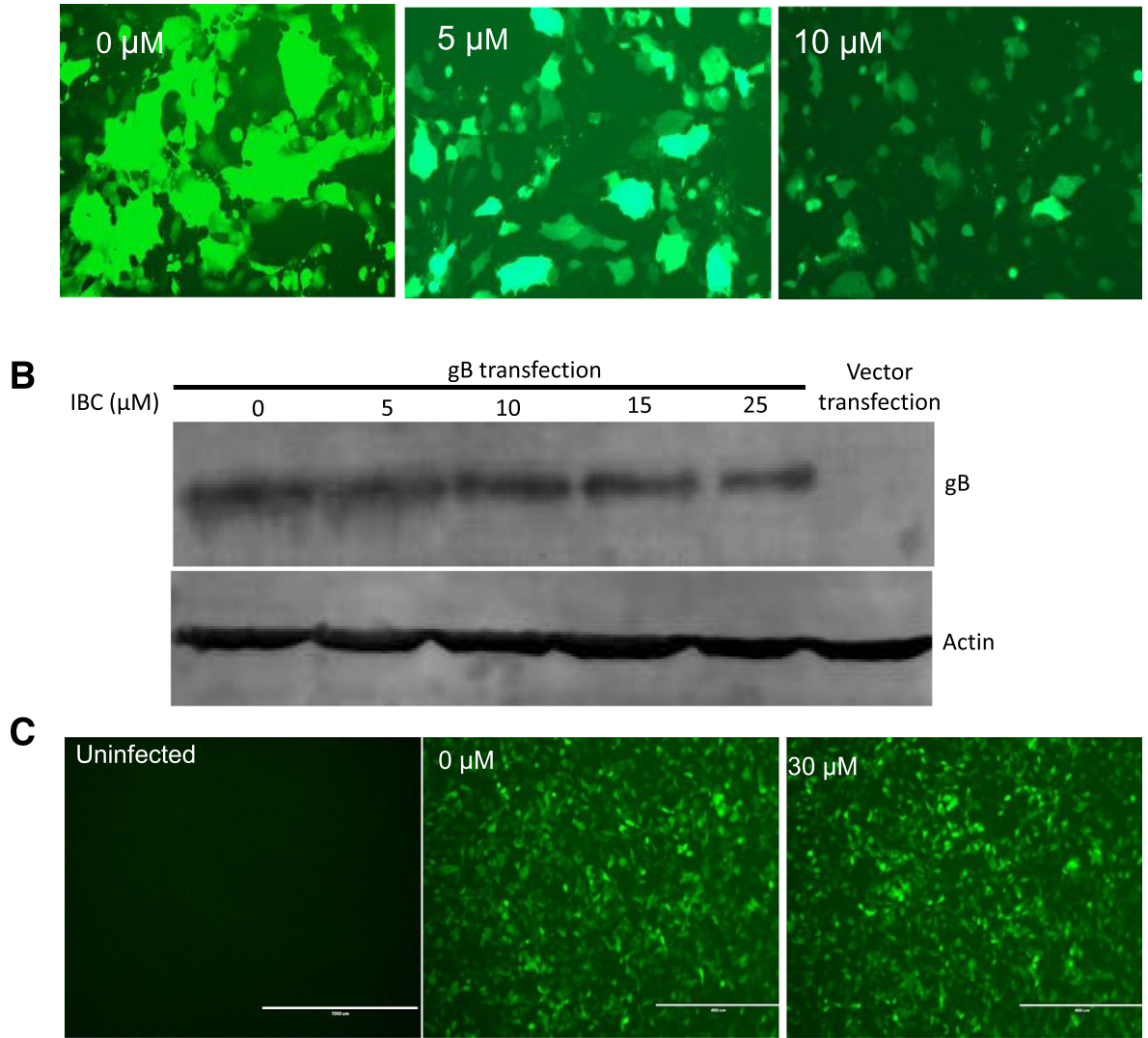

Fig. 4 IBC inhibits PRV replication at the cell-to-cell fusion step. a RK13 cells were transfected with gB, gH, gL and EGFP expression plasmids, and $6 \mathrm{~h}$ later, the medium was replaced with medium containing either ethanol or the indicated doses of IBC. Cell-to-cell fusion was analyzed by fluorescence microscopy. $\mathbf{b} \mathrm{gB}$ expression was evaluated at the indicated IBC concentrations. c Adenovirus replication was not influenced by IBC 
culture medium was replaced with medium with or without IBC, the cells were fixed $24 \mathrm{~h}$ post transfection, and syncytium formation was analyzed by fluorescence microscopy. Consistent with our hypothesis, IBC significantly inhibited PRV glycoprotein-induced cell-to-cell fusion (Fig. 4a). To exclude the possibility that the cell-to-cell fusion was caused by inhibition of gB expression by IBC, we next evaluated gB expression with a gB-specific monoclonal antibody (1:200; the gB mAb was kindly provided by Professor Zhi-Jun Tian of the Harbin Veterinary Research Institute of the Chinese Academy of Agricultural Sciences). We found that after IBC treatment, gB expression was not influenced by IBC (Fig. 4b). The above results showed that IBC inhibits PRV replication mainly at the cell-to-cell fusion step in the late stage of the lifecycle. To further confirm this finding, we evaluated IBC activity against nonenveloped adenoviruses. If IBC inhibits adenovirus replication, it may also play an antiviral role in steps of the lifecycle other than cell-to-cell fusion. An EGFP-expressing adenovirus [13] was used to infect HEK293 cells $(\mathrm{MOI}=0.1)$ in this study. Twenty-four hours later, the results indicated that adenovirus was not inhibited by IBC in HEK293 cells (Fig. 4c), further confirming that IBC inhibits PRV replication at the cell-to-cell fusion step. In our previous study, we demonstrated that the CRISPR/Cas9 system may be a powerful tool for PRV inhibition and elimination [14, 15]. However, this method requires gene transfer tools to introduce components of the CRISPR system into the cell. Thus, antiviral agents such as IBC may be more suitable for virus control in the current setting.

In conclusion, we showed that IBC exhibits antiviral activity against PRV and demonstrated that IBC treatment significantly blocked PRV-mediated cell-to-cell fusion. Therefore, IBC may be a candidate for further therapeutic evaluation against PRV infection in swine.

\section{Abbreviations}

PRV: Pseudorabies virus; GFP: Green fluorescent protein; IBC: Isobavachalcone; TCM: Traditional Chinese medicine; PRRSV: Porcine reproductive and respiratory syndrome virus

\section{Acknowledgments}

The authors would like to thank Dr. Si-Guo Liu (Harbin Veterinary Research Institute of the Chinese Academy of Agricultural Sciences) for kindly providing the $I B C$.

\section{Authors' contributions}

Y.W. T.X.L. and T.Y.W. performed the experiments. Y.-D.T and P.W. designed the experiments, analyzed the data and wrote the manuscript. The authors read and approved the final manuscript.

Funding

This study was supported by grants from the National Key R\&D Program (2016YFD0500100) and the Heilongjiang Excellent Youth Fund Project (YQ2019C028)

\section{Availability of data and materials}

Not applicable.

Ethics approval and consent to participate

Not applicable.
Consent for publication

Not applicable.

\section{Competing interests}

The authors declare that they have no competing interests.

Received: 21 January 2020 Accepted: 6 March 2020

Published online: 17 March 2020

\section{References}

1. Mettenleiter TC. Aujeszky's disease (pseudorabies) virus: the virus and molecular pathogenesis--state of the art, June 1999. Vet Res. 2000;31:99115.

2. Pomeranz LE, Reynolds AE, Hengartner CJ. Molecular biology of pseudorabies virus: impact on neurovirology and veterinary medicine. Microbiol Mol Biol Rev. 2005;69:462-500.

3. An TQ, Peng JM, Tian ZJ, Zhao HY, Li N, Liu YM, Chen JZ, Leng CL, Sun Y, Chang D, Tong GZ. Pseudorabies virus variant in Bartha-K61-vaccinated pigs, China, 2012. Emerg Infect Dis. 2013;19:1749-55.

4. Luo Y, Li N, Cong X, Wang CH, Du M, Li L, Zhao B, Yuan J, Liu DD, Li S, et al. Pathogenicity and genomic characterization of a pseudorabies virus variant isolated from Bartha-K61-vaccinated swine population in China. Vet Microbiol. 2014;174:107-15

5. Ai JW, Weng SS, Cheng O, Cui P, Li YJ, Wu HL, Zhu YM, Xu B, Zhang WH. Human Endophthalmitis caused by Pseudorabies virus infection, China, 2017. Emerg Infect Dis. 2018:24:1087-90.

6. Wong G, Lu J, Zhang W, Gao GF. Pseudorabies virus: a neglected zoonotic pathogen in humans? Emerg Microbes Infect. 2019;8:150-4

7. Yang $\mathrm{H}$, Han $\mathrm{H}$, Wang $\mathrm{H}$, Cui $Y$, Liu H, Ding $\mathrm{S}$. A case of human viral encephalitis caused by Pseudorabies virus infection in China. Front Neurol. 2019;10:534.

8. Kuete V, Sandjo LP. Isobavachalcone: an overview. Chin J Integr Med. 2012; 18:543-7.

9. Wang HM, Liu TX, Wang TY, Wang G, Liu YG, Liu SG, Tang YD, Cai XH. Isobavachalcone inhibits post-entry stages of the porcine reproductive and respiratory syndrome virus life cycle. Arch Virol. 2018;163:1263-70.

10. Tang YD, Liu JT, Fang QQ, Wang TY, Sun MX, An TQ, Tian ZJ, Cai XH. Recombinant Pseudorabies virus (PRV) expressing firefly luciferase effectively screened for CRISPR/Cas9 single guide RNAs and antiviral compounds. Viruses. 2016;8:90

11. Klupp BG, Nixdorf R, Mettenleiter TC. Pseudorabies virus glycoprotein M inhibits membrane fusion. J Virol. 2000;74:6760-8.

12. Vallbracht M, Rehwaldt S, Klupp BG, Mettenleiter TC, Fuchs W. Functional relevance of the $\mathrm{N}$-terminal domain of Pseudorabies virus envelope glycoprotein $\mathrm{H}$ and its interaction with glycoprotein L. J Virol. 2017;91: e00061-17.

13. Tang YD, Na L, Zhu CH, Shen N, Yang F, Fu XQ, Wang YH, Fu LH, Wang JY, Lin $Y Z$, et al. Equine viperin restricts equine infectious anemia virus replication by inhibiting the production and/or release of viral gag, Env, and receptor via distortion of the endoplasmic reticulum. J Virol. 2014;88:12296-310.

14. Tang YD, Guo JC, Wang TY, Zhao K, Liu JT, Gao JC, Tian ZJ, An TQ, Cai XH. CRISPR/Cas9-mediated 2-sgRNA cleavage facilitates pseudorabies virus editing. FASEB J. 2018;32:4293-301.

15. Tang YD, Liu JT, Wang TY, Sun MX, Tian ZJ, Cai XH. CRISPR/Cas9-mediated multiple single guide RNAs potently abrogate pseudorabies virus replication. Arch Virol. 2017;162:3881-6.

\section{Publisher's Note}

Springer Nature remains neutral with regard to jurisdictional claims in published maps and institutional affiliations. 